\title{
MiR-193a-5p and -3p Play a Distinct Role in Gastric Cancer: miR-193a-3p Suppresses Gastric Cancer Cell Growth by Targeting ETS1 and CCND1
}

\author{
NAN-HUA CHOU ${ }^{1 *}$, YI-HAO LO ${ }^{2 *}$, KUO-CHIANG WANG $^{1}$, CHI-HSIANG KANG ${ }^{1}$, \\ CHUNG-YU TSAI ${ }^{1}$ and KUO-WANG TSAI $3,4,5$ \\ ${ }^{1}$ Division of General Surgery, Department of Surgery, \\ Kaohsiung Veterans General Hospital, Kaohsiung, Taiwan, R.O.C.; \\ ${ }^{2}$ Department of Family Medicine, Zuoying Branch of Kaohsiung Armed \\ Forces General Hospital, Kaohsiung, Taiwan, R.O.C.; \\ ${ }^{3}$ Department of Medical Education and Research, \\ Kaohsiung Veterans General Hospital, Kaohsiung, Taiwan, R.O.C.; \\ ${ }^{4}$ Department of Chemical Biology, National Pingtung University of Education, Pingtung, Taiwan, R.O.C.; \\ ${ }^{5}$ Institute of Biomedical Sciences, National Sun Yat-Sen University, Kaohsiung, Taiwan, R.O.C.
}

\begin{abstract}
Background/Aim: MicroRNAs (miRNAs) are small non-protein-coding RNAs, that can be generated from the $5 p$ or $3 p$ arm of precursor miRNA (pre-miRNA). Differential miRNA arm selection has been reported between tumor and normal tissue in many cancer types; however, the biological function and mechanism of miRNA arm switching in gastric cancer remain unclear. Materials and Methods: Profiles of miRNA expression in gastric cancer were obtained from The Cancer Genome Atlas (TCGA). The biological role of miR$193 a-5 p /-3 p$ in tumor growth and invasive abilities was assessed through a gain-of-function approach. Target genes of miR-193a-3p were identified using bioinformatics and an experimental approach. Results: The expression levels of miR-193a-5p, and not of miR-193a-3p, were significantly decreased in gastric cancer compared to adjacent normal tissues. Ectopic expressions of miR-193a-5p and miR-193a-3p revealed that they both inhibited gastric cancer cell growth, but only miR-193a-3p significantly suppressed cell invasion ability. Using a bioinformatics approach, we identified 18 putative target genes of miR-193a-3p. Both $m R N A$ and protein levels of cyclin DI (CCNDI) and ETS proto-oncogene 1
\end{abstract}

\footnotetext{
*These Authors equally contributed to this study.

Correspondence to: Kuo-Wang Tsai, Department of Medical Education and Research, Kaohsiung Veterans General Hospital, Kaohsiung 813, Taiwan, R.O.C. Tel: +886 73422121, Fax: +886 73468056, e-mail: kwtsai6733@gmail.com
}

Key Words: miR-193a, ETS1, CCND1, gastric cancer, arm selection.
(ETS1) were significantly decreased in AGS cells transfected with miR-193a-3p mimics. ETS1 or CCND1 knockdown significantly suppressed gastric cancer cell growth, similar to miR-193a-3p overexpression. Conclusion: Our results indicated that miR-193a-3p suppressed gastric growth and motility, at least partly, by directly targeting CCND1 and ETS1 expression.

Gastric cancer is the fourth most common cancer and the second leading cause of cancer death, worldwide (1). Some of the known risk factors demonstrated to accelerate gastric cancer progression include Helicobacter pylori infection, dietary factors, tobacco use, and alcohol consumption (1). The prognosis and survival of gastric cancer patients strongly depend on stage and metastasis status. However, because of the lack of highly sensitive and specific biomarkers, gastric cancer tends to be identified at advanced stages in most patients. Gastric carcinogenesis is a complex multistep process in which DNA mutation, gene expression, and DNA epigenetic modification are quantitatively altered during cancer progression (2-5). These gene and genetic dysfunctions could be candidates as diagnostic or prognostic markers for gastric cancer $(5,6)$.

MicroRNAs (miRNAs) are small non-protein-coding RNAs; they play a crucial role in the progression of several diseases, including cancer (7). Our previous studies have reported that miRNAs-miR-9, $-34 \mathrm{~b},-129$, and $-196 \mathrm{a} / \mathrm{b}$-are suitable as biomarkers for the diagnosis or progression of gastric cancer (8-11). Mature miRNA is generated from a precursor miRNAs (pre-miRNAs), which are approximately 70 nucleotides in length and comprise a $5 \mathrm{p}$ arm, a $3 \mathrm{p}$ arm, 
and a terminal loop. An identical pre-miRNA can generate either miR-\#-5p or miR-\#-3p through Dicer processing (1214). The preference of $5 p$ or $3 p$ arm depends on the hydrogen bond theory (15). Recent studies have shown that target-mediated miRNA protection (TMMP) also contributes to miRNA arm switching $(16,17)$, leading to changes in the miRNA arm selection during the progression of cancers including gastric cancer, breast cancer, and hepatocellular carcinoma (18-20). However, the biological function and mechanism of miRNA arm switching in gastric cancer remain unclear. In this study, we identified miRNA candidates with arm switches by analyzing The Cancer Genome Atlas (TCGA) database. Furthermore, miR-193a-5p and $-3 p$ were selected for the assessment of their biological function in gastric cancer.

\section{Materials and Methods}

Expression data from the TCGA database. All level-3 expression data of breast cancer were downloaded from the TCGA database portal (https://tcga-data.nci.nih.gov/tcga/dataAccessMatrix.htm). The miRNA level-3 data of 399 gastric cancer tissues and 45 corresponding adjacent normal tissues were obtained. In addition, the level-3 data of the RNA transcriptome profile from 415 gastric cancer tissues and 35 corresponding adjacent normal tissues were obtained.

Cell lines. Human gastric cancer cell lines, AGS, were obtained from the American Type Culture Collection (ATCC, Manassas, VA, USA) and were maintained in Dulbecco's modified Eagle's medium supplemented with $10 \%$ inactivated fetal bovine serum (Invitrogen, Carlsbad, CA, USA). Total RNA was extracted using a TRIzol reagent (Invitrogen), according to the instruction manual. Samples were briefly homogenized in $1 \mathrm{ml}$ of TRIzol reagent and mixed with $0.2 \mathrm{ml}$ of chloroform to extract protein. RNA was precipitated using $0.5 \mathrm{ml}$ of isopropanol. The concentration, purity, and amount of total RNA were determined using a Nanodrop 1000 spectrophotometer (Nanodrop Technologies Inc., Wilmington, DE, USA).

Ectopic expression of miR-193a-5p and miR-193a-3p. AGS were transfected with $10 \mathrm{nM}$ of miRNA -193a-5p mimics, miR-193a-3p mimics, or the appropriate miRNA mimics control (GenDiscovery Biotechnology, Inc, Taipei, Taiwan) using Lipofectamine RNAiMAX Reagent (Invitrogen). After $24 \mathrm{~h}$ of transfection, the transfected cells were harvested, and the expression levels were examined by stem-loop for reverse transcription-quantitative realtime polymerase chain reaction (RT-qPCR).

Stem-loop $R T-q P C R$. A total of $1 \mu \mathrm{g}$ of total RNA was reversetranscribed in a stem-loop reverse transcription (RT) reaction by using RT primers and SuperScript III Reverse Transcriptase according to the user's manual (Invitrogen). The reaction was performed under the following incubation conditions: $30 \mathrm{~min}$ at $16^{\circ} \mathrm{C}$, followed by 50 cycles of $20^{\circ} \mathrm{C}$ for $30 \mathrm{~s}, 42^{\circ} \mathrm{C}$ for $30 \mathrm{~s}$, and $50^{\circ} \mathrm{C}$ for $1 \mathrm{~s}$. The enzyme was subsequently inactivated through incubation at $85^{\circ} \mathrm{C}$ for $5 \mathrm{~min}$. Real-time polymerase chain reaction (qPCR) was performed using a miR-193a-5p and miR-193a-3pspecific forward primer and a universal reverse primer. The reaction was conducted at $94^{\circ} \mathrm{C}$ for $10 \mathrm{~min}$, followed by 40 cycles of $94^{\circ} \mathrm{C}$ for $15 \mathrm{~s}$ and $60^{\circ} \mathrm{C}$ for $32 \mathrm{~s}$. The gene expression level was detected using the SYBR Green I assay (Applied Biosystems, Foster City, CA, USA), and the expression levels of miR-193a-5p or miR-193a$3 p$ were normalized to the expression of U6 non-coding small nuclear RNA. The primer sequences used to examine miRNAs are shown in Table I.

$q R T-P C R$. In total, $2 \mu \mathrm{g}$ of total RNA were reverse-transcribed with oligo (dT) 15 primers and SuperScript III Reverse Transcriptase according to the user's manual (Invitrogen). The reaction was performed with incubation at $42^{\circ} \mathrm{C}$ for $1 \mathrm{~h}$; then, the enzyme was subsequently inactivated by incubation at $85^{\circ} \mathrm{C}$ for $5 \mathrm{~min}$. The cDNA was used for the qPCR analysis with gene-specific primers, and gene expression was detected using the SYBR Green I assay (Applied Biosystems, Foster City, CA, USA). Glyceraldehyde-3phosphate dehydrogenase (GAPDH) expression was used for internal control. The sequences of ETS proto-oncogene 1(ETS1), Cyclin D1 (CCND1), and GAPDH are shown in Table I.

Western blotting. The cells were harvested $24 \mathrm{~h}$ after transient transfection; cells were washed with phosphate-buffered saline (PBS) and then lysed with lysis buffer $(50 \mathrm{mM}$ Tris- $\mathrm{HCl}$ at $\mathrm{pH} 8.0,150 \mathrm{mM}$ $\mathrm{NaCl}, 1 \% \mathrm{NP}-40,0.02 \%$ sodium azide, $1 \mu \mathrm{g} / \mathrm{mL}$ aproteinin, $1 \mathrm{mM}$ PMSF) at $4{ }^{\circ} \mathrm{C}$ for $30 \mathrm{~min}$. The lysate was collected and centrifuged to remove cell debris. Protein assays were performed using the Bio-Rad Protein Assay kit based on the Bradford dye-binding procedure (BioRad, Hercules, CA, USA). Protein samples $(60 \mu \mathrm{g})$ were separated by SDS-PAGE in $10 \%$ resolving gel using a Mini-PROTEAN 3 Cell apparatus (Bio-Rad). Proteins were then electrotransferred to polyvinylidene difluoride (PVDF) membranes (NEF1002001PK, PerkinElmer, Inc., Waltham, MA, USA). After blocking at $4^{\circ} \mathrm{C}$ overnight using PBS-Tween containing 5\% skim milk, membranes were incubated with anti-CCND1 (1:200, RM9104S, Thermo Fisher Scientific Inc., Waltham, MA, USA), anti-ETS1 (1:100, sc-55581, Santa Cruz Biotechnology, Santa Cruz, CA, USA), and anti-beta actin (ACTB; 1:2000, MAB1501, EMD Millipore, Billerica, MA, USA) for $1 \mathrm{~h}$ in PBS-Tween containing 5\% skim milk. Membranes were then incubated with anti-rabbit (sc-2004) or anti-mouse (sc-2005) IgG HRP-conjugated secondary antibodies (1:10000, Santa Cruz Biotechnology) for $1 \mathrm{~h}$ at room temperature. After three washes with PBS-Tween, immunoreactive bands were detected using ECL kit (Advansta, Menlo Park, CA, USA).

ETS1 and CCND1 knockdown with siRNA. Small interfering RNA (siRNA) oligonucleotides targeting ETS1, CCND1 and a scrambled oligo as a negative control were designed and synthesized by GenDiscovery Biotechnology (Taipei, Taiwan). The detailed information is shown in Table I. AGS cells were transfected with a final concentration $(10 \mathrm{mM})$ of individual siRNA or control using Lipofectamine RNAiMAX (Invitrogen; Thermo Fisher Scientific). After transfection for $24 \mathrm{~h}$, the protein was extracted, and knockdown efficiency was evaluated through western blotting.

Cell proliferation and invasion assay. For cell proliferation analysis, 2000 AGS cells were plated onto the 96-well plates. Cell growth was determined at $0,1,2,3$, and 4 days using 3-(4,5dimethylthiazol-2-yl)-2,5-diphenyltetrazolium bromide (MTT; Sigma-Aldrich, St. Louis, MO, USA). Cells were tested for invasion abilities in vitro in a Transwell chamber (Costar, Lowell, MA, USA). The lower side or the upper side of the polycarbonate 
Table I. Sequences of the primer sets and small interfering RNAs (siRNAS).

\begin{tabular}{ll}
\hline Primer & Primer sequences \\
\hline miR-193a-5p-RT & 5'-CTCAACTGGTGTCGTGGAGTCGGCAATT \\
& CAGTTGAGTCATCTCG-3' \\
miR-193a-5p-GSF & 5'-CGGCGGTGGGTCTTTGCGGGCG-3' \\
miR-193a-3p-RT & 5'-CTCAACTGGTGTCGTGGAGTCGGCAATT \\
& CAGTTGAGACTGGGAC -3' \\
miR-193a-3p-GSF & 5'-CGGCGGAACTGGCCTACAAAGT-3' \\
Universal reverse & 5'-CTGGTGTCGTGGAGTCGGCAATTC-3' \\
U6-F & 5'-CTCGCTTCGGCAGCACA-3' \\
U6-R & 5'-AACGCTTCACGAATTTGCGT-3' \\
ETS1-F & 5'-CTCAGATATGGAATGTGCAG-3' \\
ETS1-R & 5'-TGCTGTTCTTTAGTGAAACC-3' \\
CCND1-F & 5'-CGCCCCACCCCTCCAG-3' \\
CCND1-R & 5'-CCGCCCAGACCCTCAGACT-3' \\
GAPDH-F & 5'-TGCACCACCAACTGCTTAGC-3', \\
GAPDH-R & 5'-GGCATGGACTGTGGTCATGAG-3' \\
\hline siRNA & siRNA sequences \\
\hline si-ETS1 & sense: 5'-GCAUUAAAAGCUACUUUCATT-3' \\
si-CCND1 & antisense: 5'-UGAAAGUAGCUUUUAAUGCTT-3' \\
& sense: 5'-GGAGCAUUUUGAUACCAGATT-3' \\
& antisense: 5'-UCUGGUAUCAAAAUGCUCCGG-3'
\end{tabular}

RT, GSF, F, R

membranes (8-m pore) of the Transwell chambers were coated with $0.5 \mu \mathrm{g} / \mu \mathrm{l}$ of Matrigel and were used for invasion assays. Cells were added to the upper chamber of the Transwell chamber. After incubation for $18 \mathrm{~h}$ at $37^{\circ} \mathrm{C}$, the cells at the lower side were prepared for Giemsa staining. The level of invasion was determined using a microscope at $200 \times$ magnification. All experiments were repeated three times.

Soft agar assay. The base agar layer was prepared with $0.5 \%$ agar (Laboratorios CONDA, Torrejón de Ardoz, Madrid, Spain) dissolved in $1.5 \mathrm{ml}$ of culture medium on 6-well plates; subsequently, $1.5 \mathrm{ml}$ of $0.35 \%$ agarose (Invitrogen, Grand Island, NY, USA) solution containing the cells $(20,000$ cells/well) with CCND1 or ETS1 knockdown was inoculated on top of the base agar layer. After allowing the solution to harden, $2 \mathrm{ml}$ of fresh medium were added to the top of the hard agar layer. After 2-3 weeks, agar plates were stained with iodonitrotetrazolium chloride (SC-203739, Santa Cruz Biotechnology) and the colonies were counted.

Colony formation assay. A total of 4,000 cells were seeded on each well of a 6-well plate, and the cells were transfected with individual miR-193a-5p mimics, miR-193a-3p mimics, si-CCND1, si-ETS1, or a control using Lipofectamine RNAiMAX (Invitrogen; Thermo Fisher Scientific). After incubation at $37^{\circ} \mathrm{C}$ for 3 days, the cultured medium was replaced with a new medium. The cells were incubated at $37^{\circ} \mathrm{C}$ for 14 days. Cell culture plates containing colonies were fixed with $4 \%$ formaldehyde for $2 \mathrm{~min}$, and the colonies were stained with crystal violet solution for $2 \mathrm{~h}$. The wells were rinsed with water, and after air-drying, crystal violet stain was solubilized by adding $1 \mathrm{ml}$ of $10 \%$ acetic acid per well. The absorbance (optical density) of the solution was measured on a spectrophotometer at a wavelength of $595 \mathrm{~nm}$.

Candidate miRNA targets and assay of luciferase activity. Putative target genes of miR-193a-3p were predicted using the TargetScan tool (release no. 7.0) (21). In this study, we identified 76 candidate genes for miR-193a-3p targeting. The 3'UTR sequences of ETS1 were cloned into the pMIR-REPROT ${ }^{\mathrm{TM}}$ vector (AM5795; Thermo Fisher Scientific). Then, the pMIR-REPROT-ETS1 was cotransfected with or without miR-193a-3p mimics into gastric cancer cell line using Lipofectamine 2000 (Invitrogen). After $24 \mathrm{~h}$ of transfection, cell lysates were used for measuring luciferase activity using the Dual-Glo Luciferase Reporter Assay System (Promega, Madison, WI, USA).

Statistical analysis. The ratio of miRNA-\#-5p/miR-\#-3p between gastric cancer and adjacent normal tissue from the TCGA database were analyzed using Student's $t$-tests. The correlation of the miR\#-5p/-3p ratio between the gastric cancer and normal tissue was determined through Pearson's coefficient analysis, with $r$ and $p$ values as indicated. The expression levels of mir-193a-5p or miR$193 \mathrm{a}-3 \mathrm{p}$ in paired gastric tissues were analyzed using a paired $t$-test. The expression levels of CCND1 and ETS1 were examined in gastric cancer and adjacent normal tissues from TCGA database using Student's $t$-tests. Experiments for luciferase reporter assay, cell proliferation assay, colony formation assay, soft agar assay and invasion were conducted in triplicate. Histograms present the mean values, and the error bars indicate the standard deviation (SD). These data were analyzed using Student's $t$-tests. Differences with $p<0.05$ were considered statistically significant.

\section{Results}

Identification of miRNA arm switching using the TCGA database. TCGA database was used to analyze miRNA arm switching changes in gastric cancer. First, the small RNA-seq database, which contains 399 gastric cancers and 45 adjacent normal tissues from TCGA database, was downloaded (Figure 1A). After analyzing these miRNA profiles, 261 premiRNAs could generat both miR-\#-5p and miR-\#-3p in gastric cancer. As shown in Figure 1B, arm selection preference in most of the miRNAs was consistent $\left(\mathrm{R}^{2}=0.991\right)$ between gastric cancer and adjacent normal tissue (Figure 1B). Only a few cases were observed in which the selection of the $5 p$ and $3 p$ arm had different preferences between gastric cancer and adjacent normal tissue (fold change $>1.5$ or $<0.75 ; p<0.01$ ) (Figure $1 \mathrm{~A}$ ). The miR-\#5p/miR-\#-3p ratio of 40 miRNAs was significantly increased and that of 23 miRNAs was significantly decreased in gastric cancer compared to the adjacent normal tissues (Table II).

MiR-193a inhibits gastric cancer cell growth and motility. Among the candidates, we selected miR-193a for further study because the biological function of its $5 p$ and $3 p$ arms remains unclear in gastric cancer. As shown in Figure 1C, miR-193a-5p was significantly decreased in gastric cancer 
A

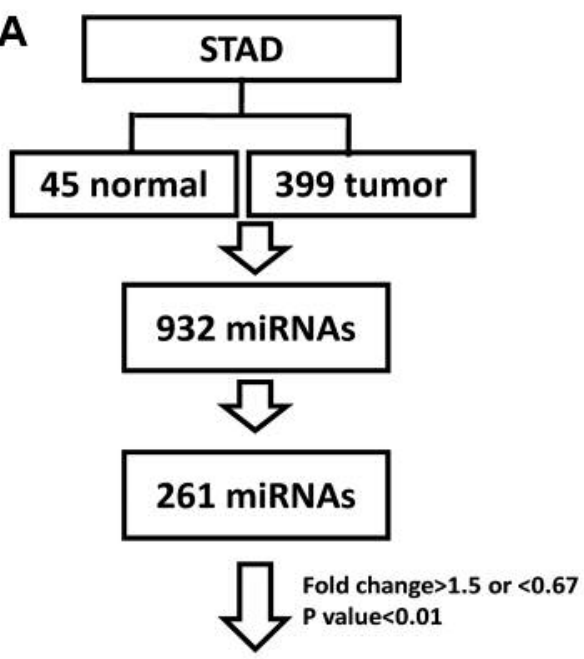

Arm switch candidates

\#63 miRNAs

C

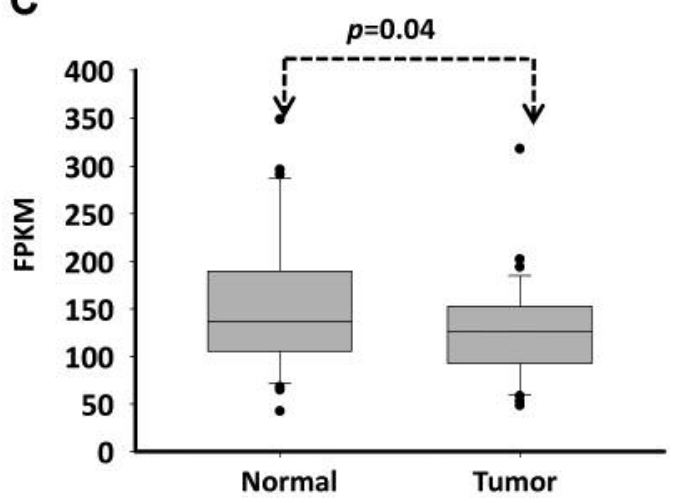

$\mathbf{E}$

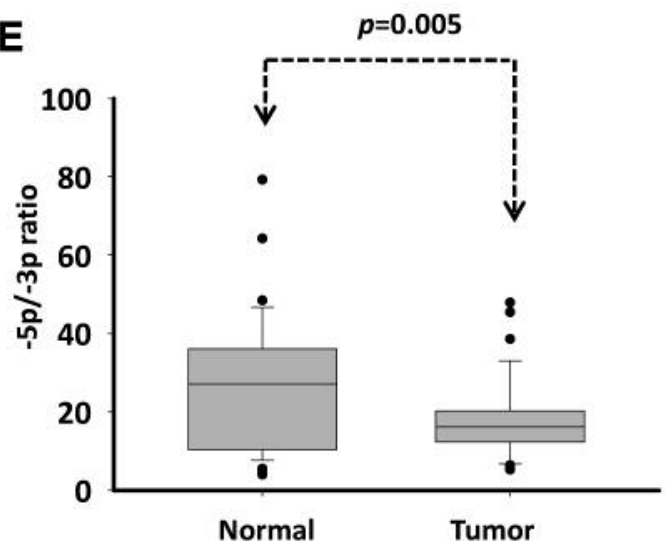

compared to the adjacent normal tissue $(p=0.04)$. No differences were observed in the expression levels of miR193a-3p between gastric cancer and adjacent normal tissue (Figure 1D). The miR-193a-5p/miR-193a-3p ratio was

B

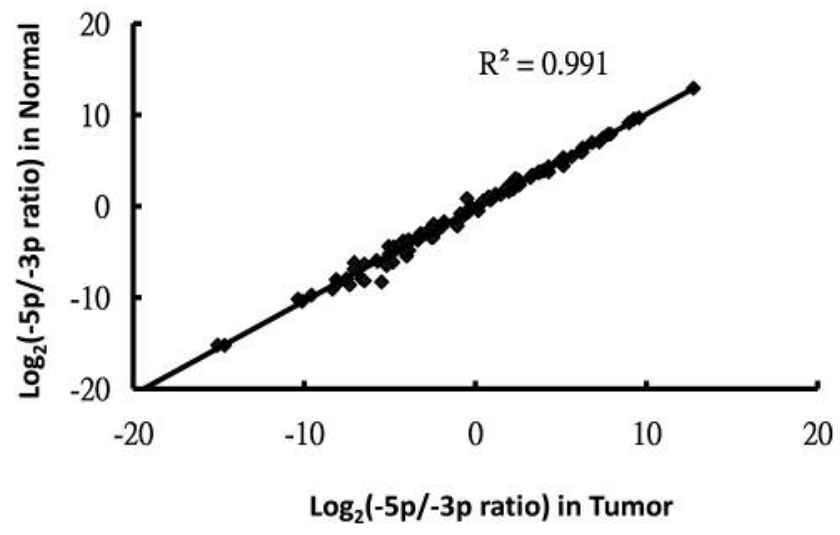

D

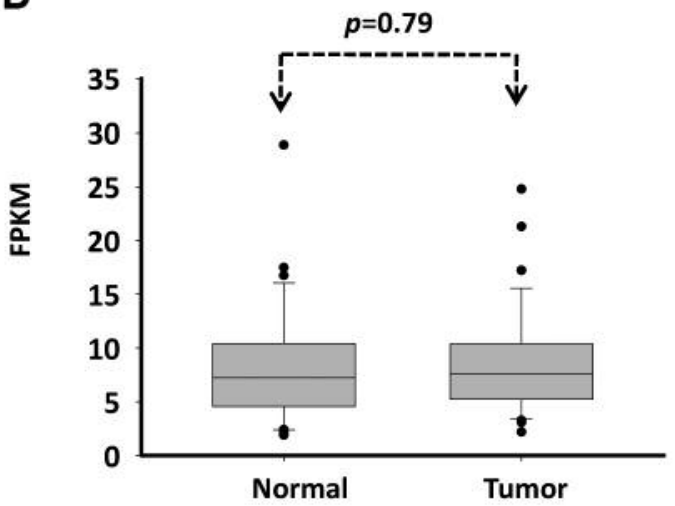

Figure 1. Identification of arm selection preference of miRNA in gastric cancer. (A): Workflow for analyzing the changes in the arm selection preference of miRNA candidates in gastric cancer. The small RNA transcriptome profiles of gastric cancer (45 normal and 399 tumor tissues) were downloaded from The Cancer Gene Atlas. The arm selection preference change candidates were selected with a significantly increased (fold change $>1.5 ; p<0.01$ ) or decreased (fold change $<0.67 ; p<0.01)$. (B): A correlation was observed in the miR-\#$5 p / m i R-\#-3 p$ ratio of 261 miRNAs between gastric cancer and normal tissues. (C-D): The expression levels of miR-193a-5p and miR-193a-3p were examined in gastric cancer and corresponding adjacent normal tissue from 37 patients. (E): The ratio of miR-193a-5p/-3p was analyzed in gastric cancer and corresponding adjacent normal tissue from 37 patients. The expression levels of the miRNA were presented in transcripts per million and analyzed using Student's $t$-test ( $p<0.05$ was considered significant). FPKM, Fragments per kilobase million.

significantly decreased in gastric cancer tissues compared to the corresponding normal tissues $(p=0.005)$ (Figure 1E), indicating changes in the arm selection preference during the miRNA maturation process. 
Table II. miRNA candidates that showed changes in arm selection preferences in gastric cancer compared to normal tissues.

Up-regulation of miR-\#-5p:-\#-3p ratio (tumor/normal)

hsa-let-7e, hsa-mir-7, hsa-mir-15a, hsa-mir-25, hsa-mir-27b, hsa-mir-29a, hsa-mir-29b, hsa-mir-30c, hsa-mir-31, hsa-mir-92a, hsa-mir-92b, hsa-mir-96, hsa-mir-105, hsa-mir-122, hsa-mir-130a, hsa-mir-135b, hsa-mir-182, hsa-mir-183, hsa-mir-188, hsa-mir-191, hsa-mir-196a, hsa-mir-196b, hsa-mir-337, hsa-mir-425, hsa-mir-431, hsa-mir-502, hsa-mir-509, hsa-mir-512, hsa-mir-517a, hsa-mir-517b, hsa-mir-519a, hsa-mir-550a, hsa-mir-654, hsa-mir-671, hsa-mir-675, hsa-mir-767, hsa-mir-877, hsa-mir-1228, hsa-mir-2116, hsa-mir-3679
Down-regulation of miR-\#-5p:-\#-3p ratio (tumor/normal)

hsa-mir-9, hsa-mir-10a, hsa-mir-10b, hsa-mir-20b, hsa-mir-26a, hsa-mir-29b, hsa-mir-99b, hsa-mir-100, hsa-mir-129, hsa-mir-193a,hsa-mir-154,hsa-mir-199a, hsa-mir-335, hsa-mir-369, hsa-mir-377, hsa-mir-423, hsa-mir-455, hsa-mir-485, hsa-mir-490, hsa-mir-491, hsa-mir-518a, hsa-mir-615, hsa-mir-1914
To investigate the biological function of the individual arms of miR-193a, miR-193a-5p and miR-193a-3p mimics were transfected into the AGS cells, respectively. Following transfection, the expressions of miR-193a-5p and $-3 p$ were significantly increased in the transfected cells compared to the scrambled controls (Figure 2A and B). Ectopic miR193a-5p slightly suppressed the proliferation and colony formation in AGS cells, and miR-193a-3p expression strongly inhibited cell growth (Figure $2 \mathrm{C}-\mathrm{E}$ ). However, only miR-193a-3p significantly inhibited the cell invasion ability (Figures 2F and 2G). These results revealed that miR-193a$5 p$ and $-3 p$ were generated from an identical pre-mir-193a; however, the arm selection preference differed significantly between gastric cancer and normal tissues. Furthermore, miR-193a-3p played crucial role in modulating the growth and invasion ability of gastric cancer cells.

Identification of target genes of miR-193a-3p in gastric cancer. We further identified putative target genes of miR193a-3p using the TargetScan tool (release no. 7.0); we found that 76 putative protein-coding genes might be regulated by miR-193a-3p (Figure 3A). Our results showed that miR-193a-3p plays a tumor suppressive role by inhibiting gastric cancer cell growth and motility; therefore, indicated that the target genes of miR-193a-3p in gastric cancer might be oncogenes. By analyzing the TCGA database, 18 gene expression levels were significantly increased in gastric cancer compared to the adjacent normal tissues. Previous studies revealed that CCND1 and ETS1 were involved in accelerating gastric cancer cell growth and invasion ability $(22,23)$. Thus, CCND1 and ETS1 were selected for further study. Our data revealed that ectopic miR-193a-3p expression significantly suppressed CCND1 and ETS1 expression both in mRNA and protein levels
(Figures 3B and 3C). The reporter assay also showed that miR-193a-3p suppressed luciferase activity by directly targeting the 3'UTR of ETS1 (Figure 3D). CCND1 and ETS1 were significantly overexpressed in gastric cancer compared to normal tissue (Figures 4A and B), and their knockdown significantly suppressed gastric cancer cell colony formation, proliferation, and anchorage-independent growth, similar to miR-193a-3p overexpression in gastric cancer cells (Figures 4C-I). In summary, our results indicated that miR-193a-3p suppressed gastric cancer cell growth by directly targeting CCND1 and ETS1 expression.

\section{Discussion}

Gastric cancer is usually diagnosed at an advanced stage and is often accompanied with metastasis, leading to poor survival. miRNAs have been reported to play a crucial role in the tumorigenesis and progression of gastric cancer $(5,6)$. In particular, miR-193a has been shown to have differential expression in gastric cancer $(24,25)$. Huang et al. reported that Linc00152 could promote gastric cancer growth by sponging miR-193a-3p expression (26). However, the detailed role of miR-193a-5p and miR-193a-3p in gastric cancer remains unknown. The present study is the first to report that both $\mathrm{miR}-193 \mathrm{a}-5 \mathrm{p}$ and $\mathrm{miR}-193 \mathrm{a}-3 \mathrm{p}$ play a tumor suppressive role in gastric cancer.

The pre-miR-193a could generate both miR-193a-5p and miR-193a-3p. We found that the ratio of the miR-193a5p/miR-193a-3p was significantly decreased in gastric cancer compared to the adjacent normal tissues. In addition, miR193a-5p, but not miR-193a-3p, was significantly decreased in gastric cancer compared to the corresponding normal tissues. Studies have reported that mature miRNA prevents degradation by interacting with its target genes $(16,17)$. 
A

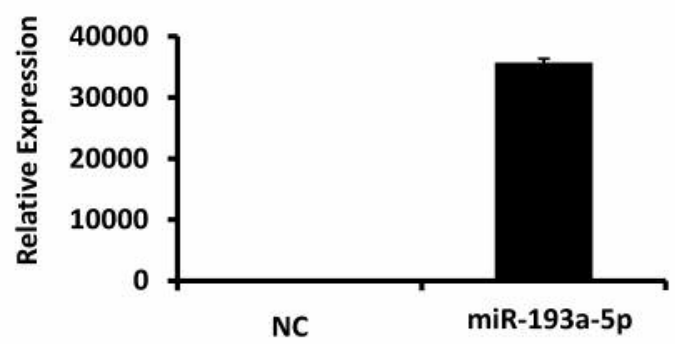

C

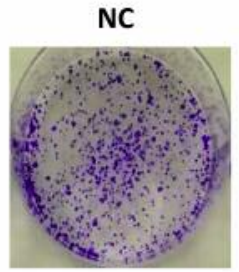

miR-193a-5p

miR-193a-3p

D

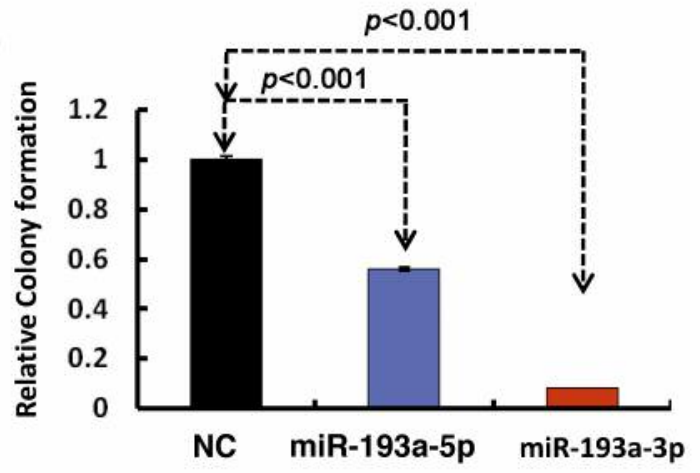

B

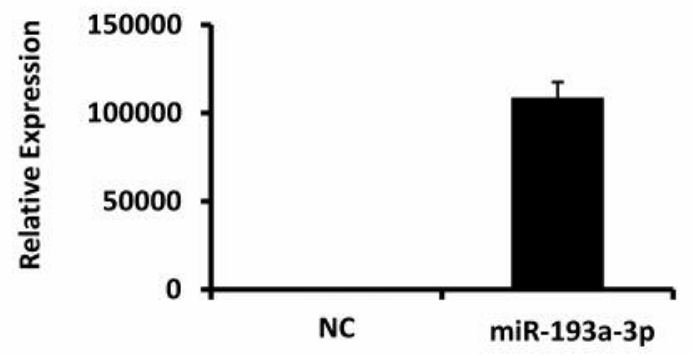

$\mathbf{E}$

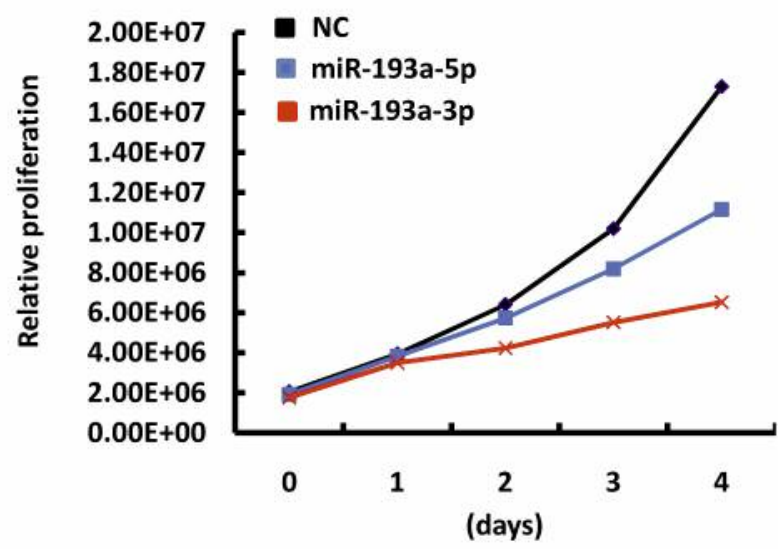

$\mathbf{F}$
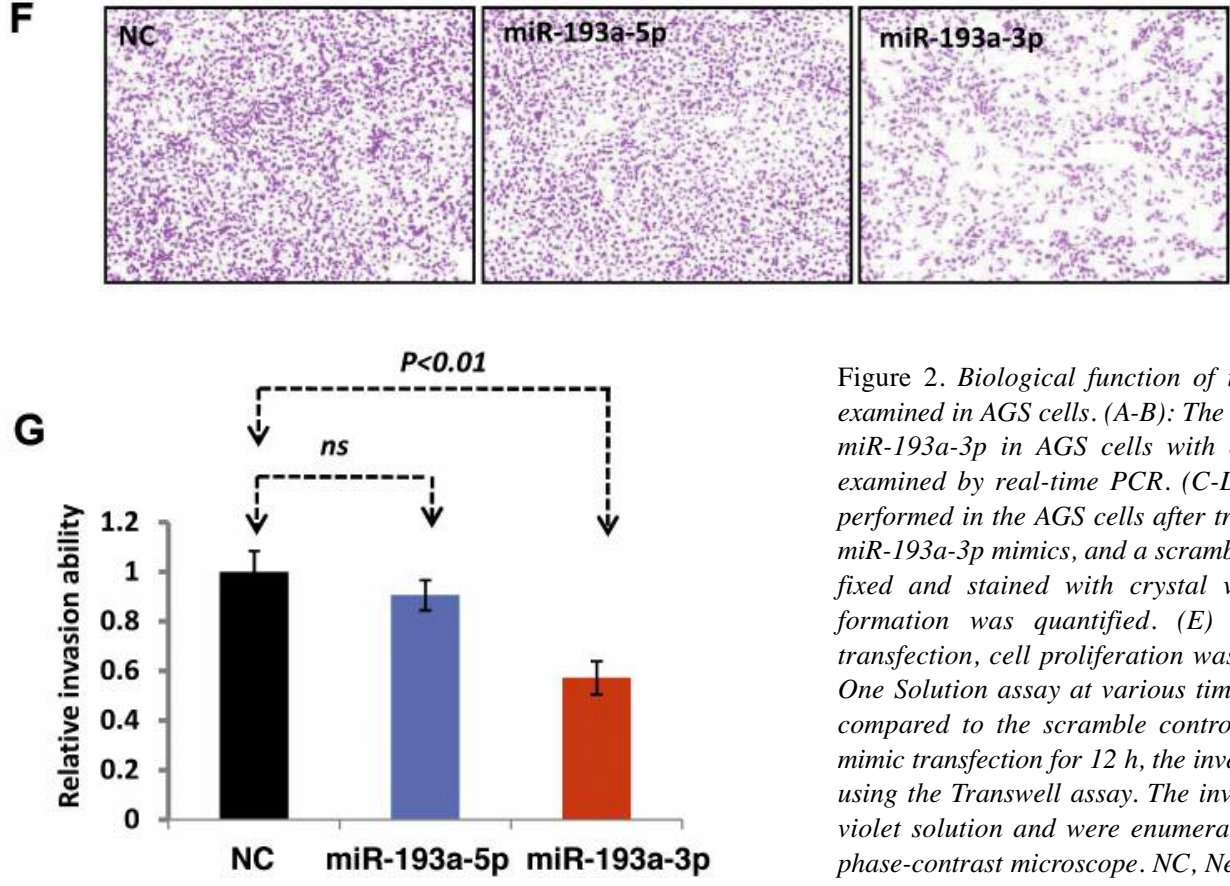

Figure 2. Biological function of the miR-193a-5p and $-3 p$ arms was examined in AGS cells. (A-B): The expression levels of miR-193a-5p and miR-193a-3p in AGS cells with or without mimic transfection were examined by real-time PCR. (C-D): The colony formation assay was performed in the AGS cells after transfection with miR-193a-5p mimics, miR-193a-3p mimics, and a scramble control for 2 weeks. The cells were fixed and stained with crystal violet solution, and relative colony formation was quantified. (E) After miR-193a-5p or $-3 p$ mimic transfection, cell proliferation was measured using the CellTiter-Glo ${ }^{\circledR}$ One Solution assay at various time points $(0,1,2,3$, and 4 days) and compared to the scramble control. (F-G): After miR-193a-5p or -3p mimic transfection for $12 \mathrm{~h}$, the invasion ability of the cells was examined using the Transwell assay. The invading cells were stained with crystal violet solution and were enumerated by counting three fields under a phase-contrast microscope. NC, Negative control; ns, non-significant. 
A

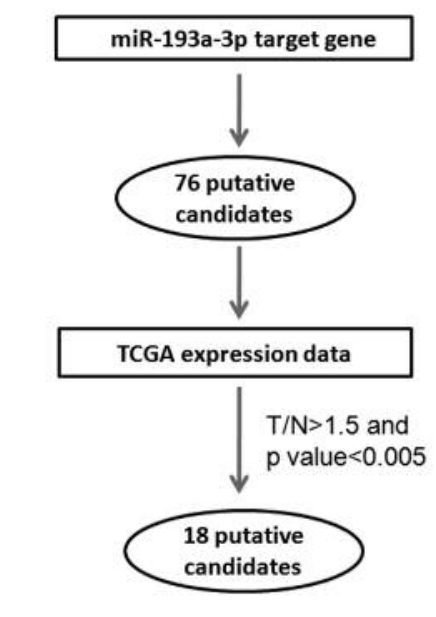

C

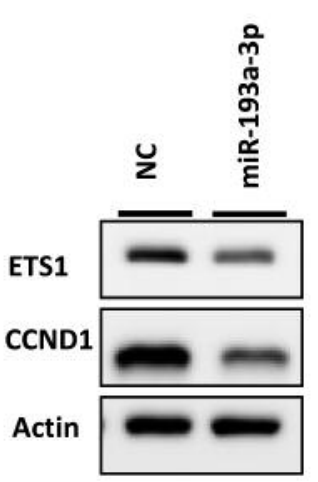

B

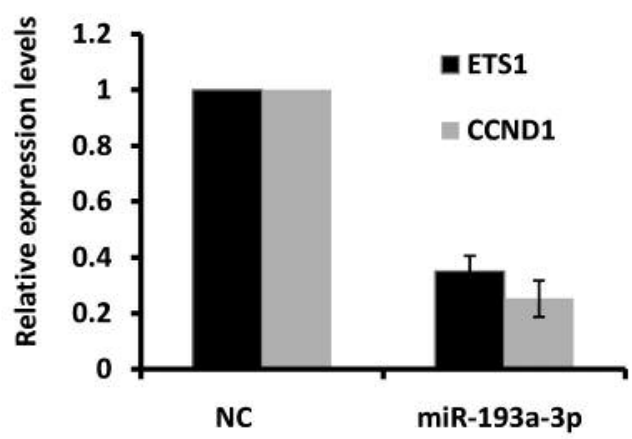

D

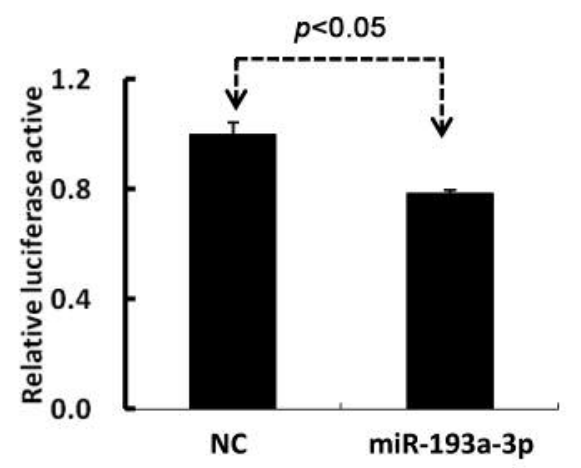

Figure 3. Identification of putative target genes of miR-193a-3p by bioinformatics and experimental approach. (A): The flowchart to identify putative target genes of miR-193a-3p by analyzing TargetScan tool and TCGA database. (B-C): The expression levels of CCND1 and ETS1 in AGS cells transfected or not with miR-193a-3p mimic were examined through real-time PCR and western blotting. (D): The luciferase reporter assay was examined in AGS cells with or without miR-193a-3p expression.

Therefore, the concept of TMMP could explain why the miR$193 a-5 p$ expression levels were decreased whereas those of miR-193a-3p were not. Taken together, these noteworthy phenomena might be due to higher number of targets of miR$193 a-3 p$ compared to miR-193a-5p in gastric cancer.

A pre-miR-193a could produce miR-193a-5p or miR193a-3p; however, the biological function might be distinct depending on their target genes. Previous studies have revealed that both miR-193a-5p and miR-193a-3p suppressed lung cancer cell migration and invasion by the co-regulation of ERBB4/PIK3R3/mTOR/S6K2 signaling pathway activity (27). They were also reported to play important roles in osteosarcoma metastasis through the inhibition of Rab27B and SRR genes (28). In the present study, functional assay showed that both miR-193a-5p and miR-193a-3p could suppress gastric cancer cell growth, but only miR-193a-3p inhibited gastric cancer cell motility. These results are consistent with those of our previous study that demonstrated that miR-193a-3p plays a crucial role in suppressing breast cancer growth and invasion (17).

Our data revealed that miR-193a-5p slightly suppressed gastric cancer cell growth, with no effect on gastric cancer invasion ability. Studies have shown that miR-193a-5p might play the opposite role in tumor development. miR-193a-5p plays the role of an oncogene in regulating cancer chemosensitivity by repressing TP73 and AP- $2 \alpha$ expression in squamous cell carcinoma, bone tumor, and bladder cancer (2931). However, others studies have revealed that miR-193a-5p plays contrasting dual functions in suppressing cancer development. Lin et al. have reported that miR-193a-5p increased radiosensitivity and suppressed the tumorigenesis of esophageal squamous cell carcinoma by directly targeting the ERBB2 expression (32). In human endometrioid endometrial adenocarcinoma, miR-193a-5p suppressed cancer cell growth and migration by modulating the miR-193a-5p-YY1-APC axis (33). Our previous study revealed that miR-193a-5p 


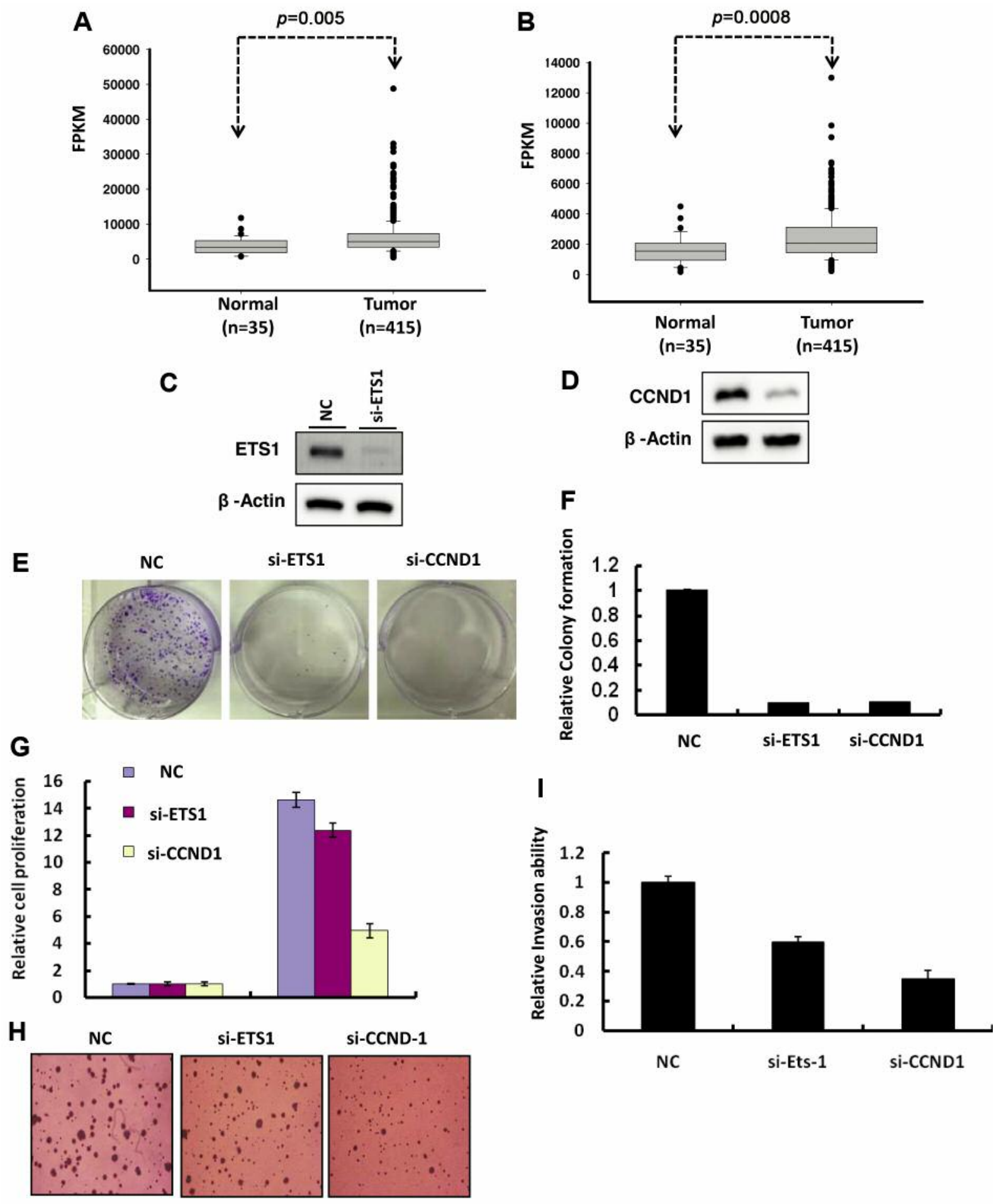

Figure 4. Biological function of CCND1 and ETS1 knockdown was examined in AGS cells. (A-B): The expression levels of CCND1 and ETS1 in gastric cancer tissues were analyzed in comparison to the corresponding adjacent normal tissues using the TCGA database. (C-D): The expression levels were examined in AGS cells transfected with small interfering RNA of CCND1 (si-CCND1), ETS1 (si-ETS1), or scramble control using western blotting. (E-F): The colony formation assay was performed in the AGS cells with si-CCND1, si-ETS1, or scramble control transfection for 2 weeks. The cells were fixed and stained with crystal violet solution, and relative colony formation was quantified. (G): After CCND1 or ETS1 knockdown, cell proliferation was measured using the CellTiter-Glo ${ }^{\circledR}$ One Solution assay at various time points ( 0 and 4 days) and was compared to the scramble control. (H-I): After CCND1 or ETS1 knockdown, the anchorage-dependent growth ability of the cells was examined using the soft agar assay. The colony was stained with iodonitrotetrazolium chloride solution and was enumerated by counting three fields under a phase-contrast microscope. FPKM, Fragments per kilobase million. 
suppressed breast cancer cell growth but did not influence the migration ability by suppressing NLN expression (17). These inconsistent results may be due to differences in cancer type. In the present study, miR-193a-5p was found to play a tumor suppressive role by slightly inhibiting gastric cancer cell growth, but the detailed mechanism remains unclear. Until now, miR-193a-3p has frequently exhibited a tumor suppressive role in human cancers $(27,34-43)$. Numerous studies have shown that miR-193a-3p could directly target oncogene expression, including uPA, MCL1, PLAU, K-Ras, CCND1, SEPN1, and IL17RD genes (17, 34-36, 42, 44, 45). In this study, we identified ETS1 as a new target of miR-193a$3 p$. Our data indicated that miR-193a-3p-induced suppression of gastric cancer cell growth might be at least partially caused by silencing of ETS1 and CCND1 expression.

In conclusion, both miR-193-5P and miR-193a-3p exert tumor-suppressing roles in gastric cancer. In particular, miR$193 a-3 p$ plays a crucial role in inhibiting gastric cancer growth and invasion ability by targeting ETS1 and CCND1 expression. These findings provide new insight into understanding the molecular mechanism underlying gastric cancer progression.

\section{Acknowledgements}

This work was supported by grants from Kaohsiung Veterans General Hospital (VGHKS-106-014 and VGHKS107-013) and Zuoying Branch of Kaohsiung Armed Forces General Hospital (ZBH-106-14 and ZBH-107-17).

\section{References}

1 Terry MB, Gaudet MM and Gammon MD: The epidemiology of gastric cancer. Semin Radiat Oncol 12: 111-127, 2002.

2 Mao Y, Zhao Q, Yin S, Ding X and Wang H: Genome-wide expression profiling and bioinformatics analysis of deregulated genes in human gastric cancer tissue after gastroscopy. Asia Pac J Clin Oncol 14: e29-e36, 2018.

3 Wang KC, Kang CH, Tsai CY, Chou NH, Tu YT, Li GC, Lam HC, Liu SI, Chang PM, Lin YH and Tsai KW: Ten-eleven translocation 1 dysfunction reduces 5-hydroxymethylcytosine expression levels in gastric cancer cells. Oncol Lett 15: 278-284, 2018.

4 Chou NH, Tsai CY, Tu YT, Wang KC, Kang CH, Chang PM, Li GC, Lam HC, Liu SI and Tsai KW: Isocitrate Dehydrogenase 2 Dysfunction Contributes to 5-hydroxymethylcytosine Depletion in Gastric Cancer Cells. Anticancer Res 36: 3983-3990, 2016.

$5 \mathrm{Wu} \mathrm{HH}$, Lin $\mathrm{WC}$ and Tsai KW: Advances in molecular biomarkers for gastric cancer: miRNAs as emerging novel cancer markers. Expert Rev Mol Med 16: e1, 2014.

6 Pan HW, Li SC and Tsai KW: MicroRNA dysregulation in gastric cancer. Curr Pharm Des 19: 1273-1284, 2013.

7 Yekta S, Shih IH and Bartel DP: MicroRNA-directed cleavage of HOXB8 mRNA. Science 304: 594-596, 2004.

8 Tsai KW, Liao YL, Wu CW, Hu LY, Li SC, Chan WC, Ho MR, Lai CH, Kao HW, Fang WL, Huang KH and Lin WC: Aberrant expression of miR-196a in gastric cancers and correlation with recurrence. Genes Chromosomes Cancer 51: 394-401, 2012.
9 Tsai KW, Wu CW, Hu LY, Li SC, Liao YL, Lai CH, Kao HW, Fang WL, Huang KH, Chan WC and Lin WC: Epigenetic regulation of miR-34b and miR-129 expression in gastric cancer. Int J Cancer 129: 2600-2610, 2011.

10 Tsai KW, Hu LY, Wu CW, Li SC, Lai CH, Kao HW, Fang WL and Lin WC: Epigenetic regulation of miR-196b expression in gastric cancer. Genes Chromosomes Cancer 49: 969-980, 2010.

11 Tsai KW, Liao YL, Wu CW, Hu LY, Li SC, Chan WC, Ho MR, Lai CH, Kao HW, Fang WL, Huang KH and Lin WC: Aberrant hypermethylation of miR-9 genes in gastric cancer. Epigenetics 6: 1189-1197, 2011.

12 Newman MA and Hammond SM: Emerging paradigms of regulated microRNA processing. Genes Dev 24: 1086-1092, 2010.

13 Trabucchi M, Briata P, Filipowicz W, Rosenfeld MG, Ramos A and Gherzi R: How to control miRNA maturation? RNA Biol 6: 536-540, 2009.

14 Slezak-Prochazka I, Durmus S, Kroesen BJ and van den Berg A: MicroRNAs, macrocontrol: regulation of miRNA processing. Rna 16: 1087-1095, 2010.

15 Rand TA, Petersen S, Du F and Wang X: Argonaute2 cleaves the anti-guide strand of siRNA during RISC activation. Cell 123: 621-629, 2005.

16 Chatterjee S, Fasler M, Bussing I and Grosshans H: Targetmediated protection of endogenous microRNAs in C. elegans. Dev Cell 20: 388-396, 2011.

17 Tsai KW, Leung CM, Lo YH, Chen TW, Chan WC, Yu SY, Tu YT, Lam HC, Li SC, Ger LP, Liu WS and Chang HT: Arm Selection Preference of MicroRNA-193a Varies in Breast Cancer. Sci Rep 6: 28176, 2016.

$18 \mathrm{Li} \mathrm{SC}$, Tsai KW, Pan HW, Jeng YM, Ho MR and Li WH: MicroRNA 3' end nucleotide modification patterns and arm selection preference in liver tissues. BMC Syst Biol 6(Suppl 2): S14, 2012.

$19 \mathrm{Li}$ SC, Liao YL, Ho MR, Tsai KW, Lai CH and Lin WC: miRNA arm selection and isomiR distribution in gastric cancer. BMC Genomics 13(Suppl 1): S13, 2012.

20 Chang HT, Li SC, Ho MR, Pan HW, Ger LP, Hu LY, Yu SY, Li WH and Tsai KW: Comprehensive analysis of microRNAs in breast cancer. BMC Genomics 13(Suppl 7): S18, 2012.

21 Lewis BP, Burge CB and Bartel DP: Conserved seed pairing, often flanked by adenosines, indicates that thousands of human genes are microRNA targets. Cell 120: 15-20, 2005.

22 Zheng L, Pu J, Qi T, Qi M, Li D, Xiang X, Huang K and Tong Q: microRNA-9 suppresses the proliferation, invasion and metastasis of gastric cancer cells through targeting cyclin D1 and Ets1. PLoS One 8: e55719, 2013.

23 Zheng L, Qi T, Yang D, Qi M, Li D, Xiang X, Huang K and Tong Q: miRNA-145 targets v-ets erythroblastosis virus E26 oncogene homolog 1 to suppress the invasion, metastasis, and angiogenesis of gastric cancer cells. Mol Cancer Res 11: 182-193, 2013.

24 Xiao J, Wang QX and Zhu YQ: Altered expression profile of micrornas in gastric stromal tumor. J Huazhong Univ Sci Technolog Med Sci 35: 842-850, 2015.

25 Wang XW, Wu Y, Wang D and Qin ZF: MicroRNA network analysis identifies key microRNAs and genes associated with precancerous lesions of gastric cancer. Genet Mol Res 13: 86958703,2014

26 Huang Y, Luo H, Li F, Yang Y, Ou G, Ye X and Li N: LINC00152 down-regulated miR-193a-3p to enhance MCL1 expression and promote gastric cancer cells proliferation. Biosci Rep pii: BSR20171607, 2018. 
27 Yu T, Li J, Yan M, Liu L, Lin H, Zhao F, Sun L, Zhang Y, Cui Y, Zhang F, Li J, He X and Yao M: MicroRNA-193a-3p and -5p suppress the metastasis of human non-small-cell lung cancer by down-regulating the ERBB4/PIK3R3/mTOR/S6K2 signaling pathway. Oncogene 34: 413-423, 2015.

$28 \mathrm{Pu}$ Y, Zhao F, Cai W, Meng X, Li Y and Cai S: MiR-193a-3p and miR-193a-5p suppress the metastasis of human osteosarcoma cells by down-regulating Rab27B and SRR, respectively. Clin Exp Metastasis 33: 359-372, 2016.

29 Jacques C, Calleja LR, Baud'huin M, Quillard T, Heymann D, Lamoureux F and Ory B: miRNA-193a-5p repression of p73 controls Cisplatin chemoresistance in primary bone tumors. Oncotarget 7: 54503-54514, 2016.

30 Zhou J, Duan H, Xie Y, Ning Y, Zhang X, Hui N, Wang C, Zhang J and Zhou J: MiR-193a-5p Targets the Coding Region of AP-2alpha mRNA and Induces Cisplatin Resistance in Bladder Cancers. J Cancer 7: 1740-1746, 2016.

31 Ory B, Ramsey MR, Wilson C, Vadysirisack DD, Forster N, Rocco JW, Rothenberg SM and Ellisen LW: A microRNA-dependent program controls p53-independent survival and chemosensitivity in human and murine squamous cell carcinoma. J Clin Invest 121: 809-820, 2011.

32 Lin $\mathrm{CH}$, Tsai CH, Yeh CT, Liang JL, Hung WC, Lin FC, Chang WL, Li HY, Yao YC, Hsu TI, Lee YC, Wang YC, Sheu BS, Lai WW, Calkins MJ, Hsiao M and Lu PJ: MiR-193a-5p/ERBB2 act as concurrent chemoradiation therapy response indicator of esophageal squamous cell carcinoma. Oncotarget 7: 39680-39693, 2016.

33 Yang Y, Zhou L, Lu L, Wang L, Li X, Jiang P, Chan LK, Zhang T, Yu J, Kwong J, Cheung TH, Chung T, Mak K, Sun H and Wang H: A novel miR-193a-5p-YY1-APC regulatory axis in human endometrioid endometrial adenocarcinoma. Oncogene 32: 34323442,2013

34 Iliopoulos D, Rotem A and Struhl K: Inhibition of miR-193a expression by Max and RXRalpha activates K-Ras and PLAU to mediate distinct aspects of cellular transformation. Cancer Res 71: 5144-5153, 2011

35 Salvi A, Conde I, Abeni E, Arici B, Grossi I, Specchia C, Portolani N, Barlati S and De Petro G: Effects of miR-193a and sorafenib on hepatocellular carcinoma cells. Mol Cancer 12: 162, 2013.

36 Wang J, Yang B, Han L, Li X, Tao H, Zhang S and Hu Y: Demethylation of miR-9-3 and miR-193a genes suppresses proliferation and promotes apoptosis in non-small cell lung cancer cell lines. Cell Physiol Biochem 32: 1707-1719, 2013.

37 Deng H, Lv L, Li Y, Zhang C, Meng F, Pu Y, Xiao J, Qian L, Zhao W, Liu Q, Zhang D, Wang Y, Zhang H, He Y and Zhu J: miR-193a-3p regulates the multi-drug resistance of bladder cancer by targeting the LOXL4 gene and the oxidative stress pathway. Mol Cancer 13: 234, 2014.
38 Lv L, Deng H, Li Y, Zhang C, Liu X, Liu Q, Zhang D, Wang L, $\mathrm{Pu}$ Y, Zhang H, He Y, Wang Y, Yu Y, Yu T and Zhu J: The DNA methylation-regulated miR-193a-3p dictates the multichemoresistance of bladder cancer via repression of SRSF2/ PLAU/HIC2 expression. Cell Death Dis 5: e1402, 2014.

39 Deng H, Lv L, Li Y, Zhang C, Meng F, Pu Y, Xiao J, Qian L, Zhao W, Liu Q, Zhang D, Wang Y, Zhang H, He Y and Zhu J: The miR-193a-3p regulated PSEN1 gene suppresses the multichemoresistance of bladder cancer. Biochim Biophys Acta 1852: 520-528, 2015.

40 Li Y, Deng H, Lv L, Zhang C, Qian L, Xiao J, Zhao W, Liu Q, Zhang D, Wang Y, Yan J, Zhang H, He Y and Zhu J: The miR193a-3p-regulated ING5 gene activates the DNA damage response pathway and inhibits multi-chemoresistance in bladder cancer. Oncotarget 6: 10195-10206, 2015.

41 Liang H, Liu M, Yan X, Zhou Y, Wang W, Wang X, Fu Z, Wang N, Zhang S, Wang Y, Zen K, Zhang CY, Hou D, Li J and Chen $\mathrm{X}$ : miR-193a-3p functions as a tumor suppressor in lung cancer by down-regulating ERBB4. J Biol Chem 290: 926-940, 2015.

42 Williams M, Kirschner MB, Cheng YY, Hanh J, Weiss J, Mugridge N, Wright CM, Linton A, Kao SC, Edelman JJ, Vallely MP, McCaughan BC, Cooper W, Klebe S, Lin RC, Brahmbhatt H, MacDiarmid J, van Zandwijk N and Reid G: miR-193a-3p is a potential tumor suppressor in malignant pleural mesothelioma. Oncotarget 6: 23480-23495, 2015.

$43 \mathrm{Lv} \mathrm{L,} \mathrm{Li} \mathrm{Y,} \mathrm{Deng} \mathrm{H,} \mathrm{Zhang} \mathrm{C,} \mathrm{Pu} \mathrm{Y,} \mathrm{Qian} \mathrm{L,} \mathrm{Xiao} \mathrm{J,} \mathrm{Zhao} \mathrm{W,}$ Liu Q, Zhang D, Wang Y, Zhang H, He Y and Zhu J: MiR-193a$3 p$ promotes the multi-chemoresistance of bladder cancer by targeting the HOXC9 gene. Cancer Lett 357: 105-113, 2015.

44 Fan Q, Hu X, Zhang H, Wang S, Zhang H, You C, Zhang CY, Liang H, Chen X and Ba Y: MiR-193a-3p is an Important Tumour Suppressor in Lung Cancer and Directly Targets KRAS. Cell Physiol Biochem 44: 1311-1324, 2017.

45 Pekow J, Meckel K, Dougherty U, Huang Y, Chen X, Almoghrabi A, Mustafi R, Ayaloglu-Butun F, Deng Z, Haider HI, Hart J, Rubin DT, Kwon JH and Bissonnette M: miR-193a$3 p$ is a Key Tumor Suppressor in Ulcerative Colitis-Associated Colon Cancer and Promotes Carcinogenesis through Upregulation of IL17RD. Clin Cancer Res 23: 5281-5291, 2017.

Received April 12, 2018

Revised May 9, 2018

Accepted May 11, 2018 